\title{
Circulating tumor cells and drug history in primary breast cancer patients
}

\author{
Silvia Jurisova ${ }^{1,2}$, Marián Karaba ${ }^{2,3}$, Gabriel Minarik ${ }^{4}$, Juraj Benca ${ }^{2,5}$, Tatiana Sedlackova ${ }^{4}$, Daniela \\ Manasova1, Katarina Kalavska1,2, Daniel Pindak ${ }^{2,3}$, Jozef Mardiak ${ }^{1,2}$, Michal Mego ${ }^{1,2}$ \\ ²nd Department of Oncology, Faculty of Medicine, Comenius University, Bratislava 83310, Slovakia. \\ ${ }^{2}$ National Cancer Institute, Bratislava 83310, Slovakia. \\ ${ }^{3}$ Slovak Medical University, Bratislava 83101, Slovakia. \\ ${ }^{4}$ Institute of Molecular Biomedicine, Faculty of Medicine, Comenius University, Bratislava 83172, Slovakia. \\ ${ }^{5}$ Department of Medicine, St. Elizabeth University, Bratislava 81250, Slovakia.
}

Correspondence to: Prof. Michal Mego, 2nd Department of Medical Oncology, Comenius University, Faculty of Medicine, National Cancer Institute, Klenova 1, Bratislava 83310, Slovak Republic. E-mail: misomego@gmail.com

How to cite this article: Jurisova S, Karaba M, Minarik G, Benca J, Sedlackova T, Manasova D, Kalavska K, Pindak D, Mardiak J, Mego M. Circulating tumor cells and drug history in primary breast cancer patients. Cancer Drug Resist 2020;3:98-109. http://dx.doi.org/10.20517/cdr.2019.79

Received: 22 Sep 2019 First Decision: 4 Dec 2019 Revised: 1 Jan 2020 Accepted: 17 Jan 2020 Published: 19 Mar 2020

Science Editor: Dario Marchetti Copy Editor: Jing-Wen Zhang Production Editor: Tian Zhang

\begin{abstract}
Aim: Different types of chronic medication may affect breast cancer prognosis. Circulating tumor cells (CTCs) play an important role in cancer metastasis formation. There is no evidence of how chronic medication affects CTCs and breast cancer prognosis. The aim of this study was to evaluate association between chronic medication and CTCs in patients with primary breast cancer.

Methods: This study involved 414 patients with stage I-III primary breast cancer. Chronic drug history was collected from patients' medical records and included all drugs that were prescribed for patients over at least the last 6 months prior to CTCs evaluation. CTCs were detected using a quantitative real-time polymerase chain reaction (qRT-PCR)-based method at the time of breast surgery.
\end{abstract}

Results: There was no association between CTCs, including their different subpopulations and chronic medication. Chronic medication using angiotensin-converting-enzyme inhibitors ( $\mathrm{ACEi}$ ), metformin, and insulin were associated with inferior disease-free survival $(\mathrm{HR}=0.49,95 \% \mathrm{Cl} 0.26-0.94, P=0.007$ for $\mathrm{ACEi} ; \mathrm{HR}=0.27,95 \% \mathrm{Cl} 0.08-0.91, P<0.001$ for metformin; and $\mathrm{HR}=0.12,95 \% \mathrm{Cl} 0.01-2.91, P<0.001$ for insulin) and this was most pronounced in patients with epithelial to mesenchymal transition (CTC_EMT) phenotype. In multivariate analysis, chronic administration of metformin and/or insulin was an independent predictor of inferior outcome.

Conclusion: Our findings show that there was no association between chronically used medication and CTCs in primary breast cancer patients. However, administration of ACEi, metformin, and/or insulin could negatively affect prognosis of patients with CTC_EMT.

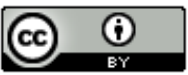

(C) The Author(s) 2020. Open Access This article is licensed under a Creative Commons Attribution 4.0 International License (https://creativecommons.org/licenses/by/4.0/), which permits unrestricted use, sharing, adaptation, distribution and reproduction in any medium or format, for any purpose, even commercially, as long as you give appropriate credit to the original author(s) and the source, provide a link to the Creative Commons license, and indicate if changes were made.

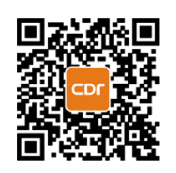


Keywords: Circulating tumor cells, chronic medication, epithelial-to-mesenchymal transition, early breast cancer

\section{INTRODUCTION}

According to global cancer statistics, breast cancer is the most commonly diagnosed cancer and the leading cause of cancer death among females, accounting for $23 \%$ of total cancer cases and $14 \%$ of cancer deaths ${ }^{[1]}$.

Circulating tumor cells (CTCs) are cancer cells intravasated into the blood stream after their separation from the primary tumor, which directly contribute to metastasis development ${ }^{[2,3]}$ and are also established as an independent predictor of progression-free and overall survival in patients with primary and metastatic breast cancer ${ }^{[4-7]}$. Although the connection between CTCs and bad prognosis is well described in breast cancer, CTCs are detected only in a subset of patients.

Comorbidities such as obesity, diabetes mellitus, hypertension, alcohol consumption, and non-cancer related drug exposure as well as regular physical activity may affect outcomes of breast cancer. Body mass index $(\mathrm{BMI}) \geq 30 \mathrm{~kg} / \mathrm{m}^{2}$ is a known factor responsible for increase in overall morbidity and mortality and is associated with breast cancer risk especially in postmenopausal women ${ }^{[8-10]}$.

Chronically used medications including non-steroidal anti-inflammatory drugs (NSAIDs), metformin, statins, and insulin may influence the progression of cancer $^{[11-14]}$. However, there is limited evidence about how chronic medication can affect CTCs. In a previous study, it was shown that patients who were treated with statins before the diagnosis of inflammatory breast cancer (IBC) had significantly lower baseline CTC counts than patients not taking statins ${ }^{[15]}$. This observation was most pronounced in patients taking $\mathrm{H}$-statins and was associated with improved progression-free and overall survival compared with nonstatin users ${ }^{[15]}$. This could be attributed to the fact that certain types of statin may block a step involved in metastasis formation, including invasion, extravasation, epithelial-mesenchymal transition, and angiogenesis, and therefore may block pathways associated with cancer stem cells ${ }^{[16]}$. However, while clinical data support this observation in IBC, there are no data about the association between CTCs and statin use in non-IBC patients ${ }^{[16]}$.

In this study, we hypothesized that certain types of chronic medication utilized before diagnosis of primary breast cancer could correlate with presence of CTCs in peripheral blood. The aim of our study was to evaluate an association between different CTC subpopulations and chronic medication and/or whether these drugs could be linked to patients' outcomes in primary breast cancer.

\section{METHODS}

\section{Study patients}

This was a prospective translational study that evaluated prognostic value of CTCs in stage I-III primary breast cancer, including 427 patients, as described previously ${ }^{[17]}$. For this sub-study, 414 patients for whom complete medical history, including drug history, was available were eligible. Chronic drug history was collected from the medical records and included all drugs that were prescribed for patient over at least the last six months before date of surgery, when CTCs were evaluated. Chronic medication was categorized into several classes including NSAIDs, $L$-thyroxin, angiotensin-converting-enzyme inhibitors (ACEi), sartans, anticoagulants (low molecular weight heparin and/or warfarin), betablockers, statins, metformin, and insulin. BMI was calculated at the time of surgery.

All study participants provided signed informed consent before study enrollment. The study was approved by the Institutional Review Board (IRB) of the National Cancer Institute of Slovakia and was conducted 
between March 2012 and February 2015. Healthy donors $(n=60)$ were age-matched women without breast cancer who were enrolled according to the IRB-approved protocol and all of them signed informed consent, as described previously ${ }^{[17]}$.

\section{Detection of CTC in peripheral blood}

Quantitative real-time polymerase chain reaction (qRT-PCR) assay was used for CTCs detection in peripheral blood that was previously depleted of CD45+ cells for CTCs enrichment, as described previously ${ }^{[17-20]}$.

\section{CTC definition}

Patient samples with higher KRT19 gene transcripts than those of healthy donors were scored as epithelial CTCs positive (CTC_EP), while patient samples with higher Epithelial-mesenchymal transition transcription factor (TWIST1, SNAIL1, SLUG, and ZEB1) gene transcripts than those of healthy donors were scored as CTC_EMT positive. Expression of at least one of the markers (either epithelial or mesenchymal) at levels above the defined cut-off was sufficient to define a sample as CTC positive ${ }^{[18,20]}$.

\section{Statistical analysis}

The patients' characteristics were summarized using the median (range) for continuous variables and frequency (percentage) for categorical variables. The median follow-up period was calculated as a median observation time among all patients and among those still alive at the time of their last follow-up. Diseasefree survival (DFS) was calculated from the date of CTC measurement to the date of disease recurrence (locoregional or distant), secondary cancer, death, or last follow-up. DFS was estimated using Kaplan-Meier product limit method and compared between groups by log-rank test. Cox-Mantel hazard ratio and 95\%CI for Cox-Mantel hazard ratio were calculated as well. Univariate analyses with Chi squared or by the Fisher's exact test were performed to find association between drug history and CTC status.

A multivariate Cox proportional hazards model for DFS was used to assess differences in outcome on the basis chronic medication, CTC_EMT status (present $v s$. absent), hormone receptor status (positive for either $v s$. negative for both), HER-2 status (positive or negative), tumor size (T1 vs. T2 and higher), axillary lymph node involvement (No vs. N+), and Ki67 status (<20\% vs. $>20 \%)$. Step-wise regression techniques were used to build multivariate models using a significance level of 0.10 to remain in the model. All $P$ values presented are two-sided, and associations were considered significant if the $P$ value is less than or equal to 0.05. Statistical analyses were performed using NCSS 11 Statistical Software (2016). NCSS, LLC. Kaysville, Utah, USA, ncss.com/software/ncss.

\section{RESULTS}

Overall, 414 patients with primary breast cancer were included in this analysis. Patients' characteristics are shown in Table 1. Median age of patients in this cohort was 60 years (range: 25-83 years). The majority of patients were of good prognosis, with tumor size less than $2 \mathrm{~cm}(69.3 \%)$, without axillary lymph nodes involvement (65.0\%), and with low/intermediate grade (65.5\%). CTC_EP was detected in 48 patients (11.6\%), while CTC_EMT in 73 patients (17.6\%); any type of CTC was present in 113 patients (27.3\%).

\section{Association between chronic medication and CTC status}

Associations between CTC and chronic medication are shown in Tables 2-4. There was no association between CTC, including different subpopulations and chronic medication, except the trend for association between CTC_EP and ACEi/sartans, where patients on ACEi/sartans had lower prevalence of CTC_EP compared to no ACEi/sartan (7.9\% vs. 16.1\%, P = 0.06). Association between BMI status and CTC was not detected [Table 5]. 
Table 1. Patients' characteristics

\begin{tabular}{|c|c|c|}
\hline & $n$ & $\%$ \\
\hline All patients & 414 & 100.0 \\
\hline \multicolumn{3}{|l|}{ T-stage } \\
\hline $\mathrm{T} 1$ & 287 & 69.3 \\
\hline$>\mathrm{T} 1$ & 127 & 30.7 \\
\hline \multicolumn{3}{|l|}{ Histology } \\
\hline IDC & 352 & 85.0 \\
\hline Other & 62 & 15.0 \\
\hline \multicolumn{3}{|l|}{ Grade } \\
\hline Low and intermediate & 271 & 65.5 \\
\hline High grade & 132 & 31.9 \\
\hline Unknown & 11 & 2.7 \\
\hline \multicolumn{3}{|l|}{ Lymph nodes } \\
\hline NO & 269 & 65.0 \\
\hline $\mathrm{N+}$ & 140 & 33.8 \\
\hline Unknown & 5 & 1.2 \\
\hline \multicolumn{3}{|c|}{ Hormone receptor status (cut-off 1\%) } \\
\hline Negative for both & 354 & 85.5 \\
\hline Positive for either & 60 & 14.5 \\
\hline \multicolumn{3}{|l|}{ HER2 status } \\
\hline Negative & 352 & 85.0 \\
\hline Positive & 62 & 15.0 \\
\hline \multicolumn{3}{|l|}{ Ki67 status (cut-off 20\%) } \\
\hline$<20 \%$ & 249 & 60.1 \\
\hline$>20 \%$ & 163 & 39.4 \\
\hline Unknown & 2 & 0.5 \\
\hline \multicolumn{3}{|l|}{ Molecular subtype } \\
\hline Luminal A & 211 & 51.0 \\
\hline Luminal B & 97 & 23.4 \\
\hline HER2+ & 62 & 15.0 \\
\hline $\mathrm{TN}$ & 42 & 10.1 \\
\hline Unknown & 2 & 0.5 \\
\hline CTC_EP & 48 & 11.6 \\
\hline CTC_EMT & 73 & 17.6 \\
\hline CTC_Any & 113 & 27.3 \\
\hline
\end{tabular}

IDC: invasive ductal carcinoma; TN: triple negative; HER2: receptor tyrosine-protein kinase erbB-2; CTC_EP: circulating tumor cell with epithelial phenotype; CTC_EMT: circulating tumor cell with epithelial-mesenchymal transition phenotype

Table 2. Association between drug history and CTC_EP

\begin{tabular}{|c|c|c|c|c|c|c|c|}
\hline \multirow{2}{*}{ Drug } & & \multirow{2}{*}{$n$} & \multicolumn{2}{|c|}{ CTC_EP negative } & \multicolumn{2}{|c|}{ CTC_EP positive } & \multirow{2}{*}{$P$-value } \\
\hline & & & $n$ & $\%$ & $n$ & $\%$ & \\
\hline \multirow[t]{2}{*}{$\overline{N S A I D}$} & No & 340 & 293 & 86.2 & 47 & 13.8 & 1.00 \\
\hline & Yes & 9 & 8 & 88.9 & 1 & 11.1 & \\
\hline \multirow[t]{2}{*}{$L$-thyroxin } & No & 307 & 263 & 85.7 & 44 & 14.3 & 0.48 \\
\hline & Yes & 42 & 38 & 90.5 & 4 & 9.5 & \\
\hline \multirow[t]{2}{*}{ ACEi } & No & 290 & 246 & 84.8 & 44 & 15.2 & 0.10 \\
\hline & Yes & 59 & 55 & 93.2 & 4 & 6.8 & \\
\hline \multirow[t]{2}{*}{ Sartans } & No & 305 & 261 & 85.6 & 44 & 14.4 & 0.48 \\
\hline & Yes & 44 & 40 & 90.9 & 4 & 9.1 & \\
\hline \multirow[t]{2}{*}{ ACEi/Sartan } & No & 248 & 208 & 83.9 & 40 & 16.1 & 0.06 \\
\hline & Yes & 101 & 93 & 92.1 & 8 & 7.9 & \\
\hline \multirow[t]{2}{*}{ Betablockers } & No & 250 & 218 & 87.2 & 32 & 12.8 & 0.40 \\
\hline & Yes & 99 & 83 & 83.8 & 16 & 16.2 & \\
\hline \multirow[t]{2}{*}{ Statins } & No & 295 & 251 & 85.1 & 44 & 14.9 & 0.20 \\
\hline & Yes & 54 & 50 & 92.6 & 4 & 7.4 & \\
\hline \multirow[t]{2}{*}{ Metformin } & No & 332 & 285 & 85.8 & 47 & 14.2 & 0.49 \\
\hline & Yes & 17 & 16 & 94.1 & 1 & 5.9 & \\
\hline Insulin & No & 345 & 297 & 86.1 & 48 & 13.9 & 1.00 \\
\hline
\end{tabular}


Page 102 Jurisova et al. Cancer Drug Resist 2020;3:98-109 I http://dx.doi.org/10.20517/cdr.2019.79

\begin{tabular}{llllllll} 
& Yes & 4 & 4 & 100.0 & 0 & 0.0 & \\
Insulin/Metformin & No & 329 & 282 & 85.7 & 47 & 14.3 & 0.33 \\
& Yes & 20 & 19 & 95.0 & 1 & 5.0 & \\
LMWH/Warfarin & No & 335 & 288 & 86.0 & 47 & 14.0 & 0.70 \\
& Yes & 14 & 13 & 92.9 & 1 & 7.1 & \\
\hline
\end{tabular}

NSAID: non-steroidal anti-inflammatory drugs; ACEi: angiotensin-converting-enzyme inhibitors; LMWH: low molecular weight heparin; CTC_EP: circulating tumor cell with epithelial phenotype

Table 3. Association between drug history and CTC_EMT

\begin{tabular}{|c|c|c|c|c|c|c|c|}
\hline \multirow{2}{*}{ Drug } & & \multirow{2}{*}{$n$} & \multicolumn{2}{|c|}{ CTC_EMT negative } & \multicolumn{2}{|c|}{ CTC_EMT positive } & \multirow{2}{*}{$P$-value } \\
\hline & & & $n$ & $\%$ & $n$ & $\%$ & \\
\hline \multirow[t]{2}{*}{ NSAID } & No & 366 & 293 & 80.1 & 73 & 19.9 & 0.36 \\
\hline & Yes & 8 & 8 & 100.0 & 0 & 0.0 & \\
\hline \multirow[t]{2}{*}{$L$-thyroxin } & No & 325 & 263 & 80.9 & 62 & 19.1 & 0.57 \\
\hline & Yes & 49 & 38 & 77.6 & 11 & 22.4 & \\
\hline \multirow[t]{2}{*}{ ACEi } & No & 306 & 246 & 80.4 & 60 & 19.6 & 1.00 \\
\hline & Yes & 68 & 55 & 80.9 & 13 & 19.1 & \\
\hline \multirow[t]{2}{*}{ Sartans } & No & 319 & 261 & 81.8 & 58 & 18.2 & 0.14 \\
\hline & Yes & 55 & 40 & 72.7 & 15 & 27.3 & \\
\hline \multirow[t]{2}{*}{ ACEi/Sartan } & No & 254 & 208 & 81.9 & 46 & 18.1 & 0.33 \\
\hline & Yes & 120 & 93 & 77.5 & 27 & 22.5 & \\
\hline \multirow[t]{2}{*}{ Betablockers } & No & 267 & 218 & 81.6 & 49 & 18.4 & 0.39 \\
\hline & Yes & 107 & 83 & 77.6 & 24 & 22.4 & \\
\hline \multirow[t]{2}{*}{ Statins } & No & 312 & 251 & 80.4 & 61 & 19.6 & 1.00 \\
\hline & Yes & 62 & 50 & 80.6 & 12 & 19.4 & \\
\hline \multirow[t]{2}{*}{ Metformin } & No & 352 & 285 & 81.0 & 67 & 19.0 & 0.40 \\
\hline & Yes & 22 & 16 & 72.7 & 6 & 27.3 & \\
\hline \multirow[t]{2}{*}{ Insulin } & No & 370 & 297 & 80.3 & 73 & 19.7 & 1.00 \\
\hline & Yes & 4 & 4 & 100.0 & 0 & 0.0 & \\
\hline \multirow[t]{2}{*}{ Insulin/Metformin } & No & 349 & 282 & 80.8 & 67 & 19.2 & 0.60 \\
\hline & Yes & 25 & 19 & 76.0 & 6 & 24.0 & \\
\hline \multirow[t]{2}{*}{ LMWH/Warfarin } & No & 358 & 288 & 80.4 & 70 & 19.6 & 1.00 \\
\hline & Yes & 16 & 13 & 81.3 & 3 & 18.8 & \\
\hline
\end{tabular}

NSAID: non-steroidal anti-inflammatory drugs; ACEi: angiotensin-converting-enzyme inhibitors; LMWH: low molecular weight heparin; CTC_EMT: circulating tumor cell with epithelial-mesenchymal transition phenotype

Table 4. Association between drug history and CTC

\begin{tabular}{|c|c|c|c|c|c|c|c|}
\hline \multirow{2}{*}{ Drug } & & \multirow{2}{*}{$n$} & \multicolumn{2}{|c|}{ CTC_Any negative } & \multicolumn{2}{|c|}{ CTC_Any positive } & \multirow{2}{*}{$P$-value } \\
\hline & & & $n$ & $\%$ & $n$ & $\%$ & \\
\hline \multirow[t]{2}{*}{ NSAID } & No & 405 & 293 & 72.3 & 112 & 27.7 & 0.45 \\
\hline & Yes & 9 & 8 & 88.9 & 1 & 11.1 & \\
\hline \multirow[t]{2}{*}{$L$-thyroxin } & No & 362 & 263 & 72.7 & 99 & 27.3 & 1.00 \\
\hline & Yes & 52 & 38 & 73.1 & 14 & 26.9 & \\
\hline \multirow[t]{2}{*}{ ACEi } & No & 343 & 246 & 71.7 & 97 & 28.3 & 0.38 \\
\hline & Yes & 71 & 55 & 77.5 & 16 & 22.5 & \\
\hline \multirow[t]{2}{*}{ Sartans } & No & 355 & 261 & 73.5 & 94 & 26.5 & 0.35 \\
\hline & Yes & 59 & 40 & 67.8 & 19 & 32.2 & \\
\hline \multirow[t]{2}{*}{ ACEi/Sartan } & No & 287 & 208 & 72.5 & 79 & 27.5 & 0.91 \\
\hline & Yes & 127 & 93 & 73.2 & 34 & 26.8 & \\
\hline \multirow[t]{2}{*}{ Betablockers } & No & 296 & 218 & 73.6 & 78 & 26.4 & 0.54 \\
\hline & Yes & 118 & 83 & 70.3 & 35 & 29.7 & \\
\hline \multirow[t]{2}{*}{ Statins } & No & 350 & 251 & 71.7 & 99 & 28.3 & 0.36 \\
\hline & Yes & 64 & 50 & 78.1 & 14 & 21.9 & \\
\hline \multirow[t]{2}{*}{ Metformin } & No & 392 & 285 & 72.7 & 107 & 27.3 & 1.00 \\
\hline & Yes & 22 & 16 & 72.7 & 6 & 27.3 & \\
\hline Insulin & No & 410 & 297 & 72.4 & 113 & 27.6 & 0.58 \\
\hline
\end{tabular}




\begin{tabular}{llllllll} 
& Yes & 4 & 4 & 100.0 & 0 & 0.0 & \\
Insulin/Metformin & No & 389 & 282 & 72.5 & 107 & 27.5 & 0.82 \\
& Yes & 25 & 19 & 76.0 & 6 & 24.0 & \\
LMWH/Warfarin & No & 397 & 288 & 72.5 & 109 & 27.5 & 1.00 \\
& Yes & 17 & 13 & 76.5 & 4 & 23.5 & \\
\hline
\end{tabular}

NSAID: non-steroidal anti-inflammatory drugs; ACEi: angiotensin-converting-enzyme inhibitors; LMWH: low molecular weight heparin; CTC: circulating tumor cell

Table 5. Association between BMI and CTC

\begin{tabular}{|c|c|c|c|c|c|c|}
\hline \multirow{2}{*}{ BMI } & \multirow{2}{*}{$n$} & \multicolumn{2}{|c|}{ CTC_EP negative } & \multicolumn{2}{|c|}{ CTC_EP positive } & \multirow{2}{*}{$\boldsymbol{P}$-value } \\
\hline & & $n$ & $\%$ & $n$ & $\%$ & \\
\hline$<25$ & 180 & 157 & 87.2 & 23 & 12.8 & 0.47 \\
\hline $26-30$ & 118 & 107 & 90.7 & 11 & 9.3 & \\
\hline $31-35$ & 92 & 80 & 87.0 & 12 & 13.0 & \\
\hline \multirow[t]{2}{*}{$>36$} & 23 & 22 & 95.7 & 1 & 4.3 & \\
\hline & & \multicolumn{2}{|c|}{ CTC_EMT negative } & \multicolumn{2}{|c|}{ CTC_EMT positive } & $P$-value \\
\hline$<25$ & 180 & 150 & 83.3 & 30 & 16.7 & 0.46 \\
\hline $26-30$ & 118 & 99 & 83.9 & 19 & 16.1 & \\
\hline $31-35$ & 92 & 75 & 81.5 & 17 & 18.5 & \\
\hline \multirow[t]{2}{*}{$>36$} & 23 & 16 & 69.6 & 7 & 30.4 & \\
\hline & & \multicolumn{2}{|c|}{ CTC_Any negative } & \multicolumn{2}{|c|}{ CTC_Any positive } & $P$-value \\
\hline$<25$ & 180 & 132 & 73.3 & 48 & 26.7 & 0.60 \\
\hline $26-30$ & 118 & 90 & 76.3 & 28 & 23.7 & \\
\hline $31-35$ & 92 & 64 & 69.6 & 28 & 30.4 & \\
\hline$>36$ & 23 & 15 & 65.2 & 8 & 34.8 & \\
\hline
\end{tabular}

BMI: body mass index; CTC: circulating tumor cell; CTC_EP: circulating tumor cell with epithelial phenotype; CTC_EMT: circulating tumor cell with epithelial-mesenchymal transition phenotype

Table 6. Impact of drug history on disease-free survival in primary breast cancer

\begin{tabular}{|c|c|c|c|c|c|}
\hline Drug & $n$ & $\mathrm{HR}^{\star}$ & 95\% Low ${ }^{\star \star}$ & 95\% High ${ }^{\star \star}$ & $P$-value ${ }^{\star \star \star}$ \\
\hline $\begin{array}{l}\text { No NSAID } \\
\text { NSAID }\end{array}$ & $\begin{array}{l}405 \\
9\end{array}$ & 0.00 & 0.00 & 0.00 & 0.200 \\
\hline $\begin{array}{l}\text { No } L \text {-thyroxin } \\
L \text {-thyroxin }\end{array}$ & $\begin{array}{l}362 \\
52\end{array}$ & 0.93 & 0.45 & 1.91 & 0.840 \\
\hline $\begin{array}{l}\text { No ACEi } \\
\text { ACEi }\end{array}$ & $\begin{array}{l}343 \\
71\end{array}$ & 0.49 & 0.26 & 0.94 & 0.007 \\
\hline $\begin{array}{l}\text { No sartans } \\
\text { Sartans }\end{array}$ & $\begin{array}{l}355 \\
59\end{array}$ & 0.69 & 0.35 & 1.37 & 0.230 \\
\hline $\begin{array}{l}\text { No ACEi/sartan } \\
\text { ACEi/sartan }\end{array}$ & $\begin{array}{l}287 \\
127\end{array}$ & 0.53 & 0.31 & 0.89 & 0.008 \\
\hline $\begin{array}{l}\text { No betablockers } \\
\text { Betablockers }\end{array}$ & $\begin{array}{l}296 \\
118\end{array}$ & 0.82 & 0.49 & 1.40 & 0.450 \\
\hline $\begin{array}{l}\text { No statins } \\
\text { Statins }\end{array}$ & $\begin{array}{l}350 \\
64\end{array}$ & 0.63 & 0.32 & 1.22 & 0.110 \\
\hline $\begin{array}{l}\text { No metformin } \\
\text { Metformin }\end{array}$ & $\begin{array}{l}392 \\
22\end{array}$ & 0.27 & 0.08 & 0.91 & $<0.001$ \\
\hline $\begin{array}{l}\text { No insulin } \\
\text { Insulin }\end{array}$ & $\begin{array}{l}410 \\
4\end{array}$ & 0.12 & 0.01 & 2.91 & $<0.001$ \\
\hline $\begin{array}{l}\text { No insulin/metformin } \\
\text { Insulin/metformin }\end{array}$ & $\begin{array}{l}389 \\
25\end{array}$ & 0.24 & 0.08 & 0.77 & $<0.001$ \\
\hline $\begin{array}{l}\text { No LMWH/warfarin } \\
\text { LMWH/warfarin }\end{array}$ & $\begin{array}{l}397 \\
17\end{array}$ & 1.43 & 0.43 & 4.71 & 0.620 \\
\hline
\end{tabular}

${ }^{\star}$ Cox-Mantel hazard ratio; ${ }^{\star *} 95 \% \mathrm{Cl}$ for Cox-Mantel hazard ratio; ${ }^{\star \star \star}$ equal-weighted logrank test. HR: hazard ratio; NSAID: non-steroidal anti-inflammatory drugs; ACEi: angiotensin-converting-enzyme inhibitors; LMWH: low molecular weight heparin

\section{Disease outcome according to chronic medication}

At a median follow-up time of 55.0 months (range: 4.9-76.7 months), 74 patients (17.3\%) had experienced a DFS event, and 36 patients $(8.4 \%)$ had died. In univariate analysis, chronic administration of ACEi, 
Table 7. Impact of drug history on disease-free survival in primary breast cancer according to CTC subgroups

\begin{tabular}{|c|c|c|c|c|c|}
\hline & Sample size 0/1 & $\mathbf{H R}^{\star}$ & 95\% Low ${ }^{\star \star}$ & 95\% High ${ }^{\star \star}$ & $P$-value ${ }^{\star \star \star}$ \\
\hline \multicolumn{6}{|l|}{ No NSAID vs. NSAID } \\
\hline CTC negative & $293 / 8$ & 0.00 & 0.00 & 0.00 & 0.253 \\
\hline CTC_EP positive & $47 / 1$ & 0.00 & 0.00 & 0.00 & 0.739 \\
\hline CTC_EMT positive & NA & NA & NA & NA & NA \\
\hline CTC_any & $112 / 1$ & 0.00 & 0.00 & 0.00 & 0.673 \\
\hline \multicolumn{6}{|c|}{ No $L$-thyroxin $v s$. t-thyroxin } \\
\hline CTC negative & $263 / 38$ & 0.89 & 0.36 & 2.18 & 0.782 \\
\hline CTC_EP positive & $44 / 4$ & 0.30 & 0.01 & 9.38 & 0.251 \\
\hline CTC_EMT positive & $62 / 11$ & 1.18 & 0.37 & 3.77 & 0.785 \\
\hline CTC_any & $99 / 14$ & 1.10 & 0.34 & 3.54 & 0.874 \\
\hline \multicolumn{6}{|l|}{ No ACEi vs. ACEi } \\
\hline CTC negative & $246 / 55$ & 0.58 & 0.27 & 1.26 & 0.104 \\
\hline CTC_EP positive & $44 / 4$ & 0.36 & 0.01 & 8.7 & 0.339 \\
\hline CTC_EMT positive & $60 / 13$ & 0.36 & 0.1 & 1.31 & 0.029 \\
\hline CTC_any & $97 / 16$ & 0.27 & 0.07 & 1.03 & 0.002 \\
\hline \multicolumn{6}{|l|}{ No sartan vs. sartan } \\
\hline CTC negative & $261 / 40$ & 0.67 & 0.28 & 1.62 & 0.308 \\
\hline CTC_EP positive & $44 / 4$ & 0.00 & 0.00 & 0.00 & 0.490 \\
\hline CTC_EMT positive & $58 / 15$ & 0.78 & 0.27 & 2.31 & 0.636 \\
\hline CTC_any & $94 / 19$ & 0.79 & 0.27 & 2.28 & 0.642 \\
\hline \multicolumn{6}{|c|}{ No ACEi/sartan vs. ACEi/sartan } \\
\hline CTC negative & $208 / 93$ & 0.57 & 0.3 & 1.09 & 0.063 \\
\hline CTC_EP positive & $40 / 8$ & 0.82 & 0.08 & 8.44 & 0.856 \\
\hline CTC_EMT positive & $46 / 27$ & 0.50 & 0.20 & 1.27 & 0.114 \\
\hline CTC_any & $79 / 34$ & 0.43 & 0.18 & 1.07 & 0.036 \\
\hline \multicolumn{6}{|c|}{ No blockers vs. blockers } \\
\hline CTC negative & $218 / 83$ & 0.93 & 0.48 & 1.79 & 0.817 \\
\hline CTC_EP positive & $32 / 16$ & 1.98 & 0.31 & 12.73 & 0.535 \\
\hline CTC_EMT positive & $49 / 24$ & 0.51 & 0.2 & 1.34 & 0.131 \\
\hline CTC_any & $78 / 35$ & 0.72 & 0.3 & 1.72 & 0.431 \\
\hline \multicolumn{6}{|l|}{ No statin vs. statin } \\
\hline CTC negative & $251 / 50$ & 0.53 & 0.24 & 1.17 & 0.054 \\
\hline CTC_EP positive & $44 / 4$ & 0.00 & 0.00 & 0.00 & 0.490 \\
\hline CTC_EMT positive & $61 / 12$ & 0.92 & 0.26 & 3.26 & 0.892 \\
\hline CTC_any & $99 / 14$ & 0.85 & 0.23 & 3.07 & 0.787 \\
\hline \multicolumn{6}{|c|}{ No metformin vs. metformin } \\
\hline CTC negative & $285 / 16$ & 0.37 & 0.09 & 1.53 & 0.027 \\
\hline CTC_EP positive & $47 / 1$ & 0.00 & 0.00 & 0.00 & 0.739 \\
\hline CTC_EMT positive & $67 / 6$ & 0.13 & 0.01 & 1.6 & $<0.001$ \\
\hline CTC_any & $107 / 6$ & 0.10 & 0.01 & 1.78 & $<0.001$ \\
\hline \multicolumn{6}{|l|}{ No Insulin vs. Insulin } \\
\hline СТC negative & $297 / 4$ & 0.11 & 0.00 & 3.00 & $<0.001$ \\
\hline CTC_EP positive & NA & NA & NA & NA & NA \\
\hline CTC_EMT positive & NA & NA & NA & NA & NA \\
\hline CTC_any & NA & NA & NA & NA & NA \\
\hline \multicolumn{6}{|c|}{ No Insulin/metformin vs. Insulin/metformin } \\
\hline CTC negative & $282 / 19$ & 0.29 & 0.08 & 1.1 & 0.002 \\
\hline CTC_EP positive & $47 / 1$ & 0.00 & 0.00 & 0.00 & 0.739 \\
\hline CTC_EMT positive & $67 / 6$ & 0.13 & 0.01 & 1.6 & $<0.001$ \\
\hline CTC_any & $107 / 6$ & 0.1 & 0.01 & 1.78 & $<0.001$ \\
\hline \multicolumn{6}{|l|}{ No LMWH vs. LMWH } \\
\hline CTC negative & $288 / 13$ & 0.96 & 0.23 & 4.08 & 0.960 \\
\hline CTC_EP positive & $47 / 1$ & 0.00 & 0.00 & 0.00 & 0.739 \\
\hline CTC_EMT positive & $70 / 3$ & 0.00 & 0.00 & 0.00 & 0.312 \\
\hline CTC_any & $109 / 4$ & 0.00 & 0.00 & 0.00 & 0.348 \\
\hline
\end{tabular}

${ }^{\star}$ Cox-Mantel hazard ratio; ${ }^{\star *} 95 \% \mathrm{Cl}$ for Cox-Mantel hazard ratio; ${ }^{* \star \star}$ equal-weighted logrank test. NSAID: non-steroidal anti-inflammatory drugs; ACEi: angiotensin-converting-enzyme inhibitors; LMWH: low molecular weight heparin; CTC: circulating tumor cell; CTC_EP: circulating tumor cell with epithelial phenotype; CTC_EMT: circulating tumor cell with epithelial-mesenchymal transition phenotype; HR: hazard ratio; NA: not applicable 


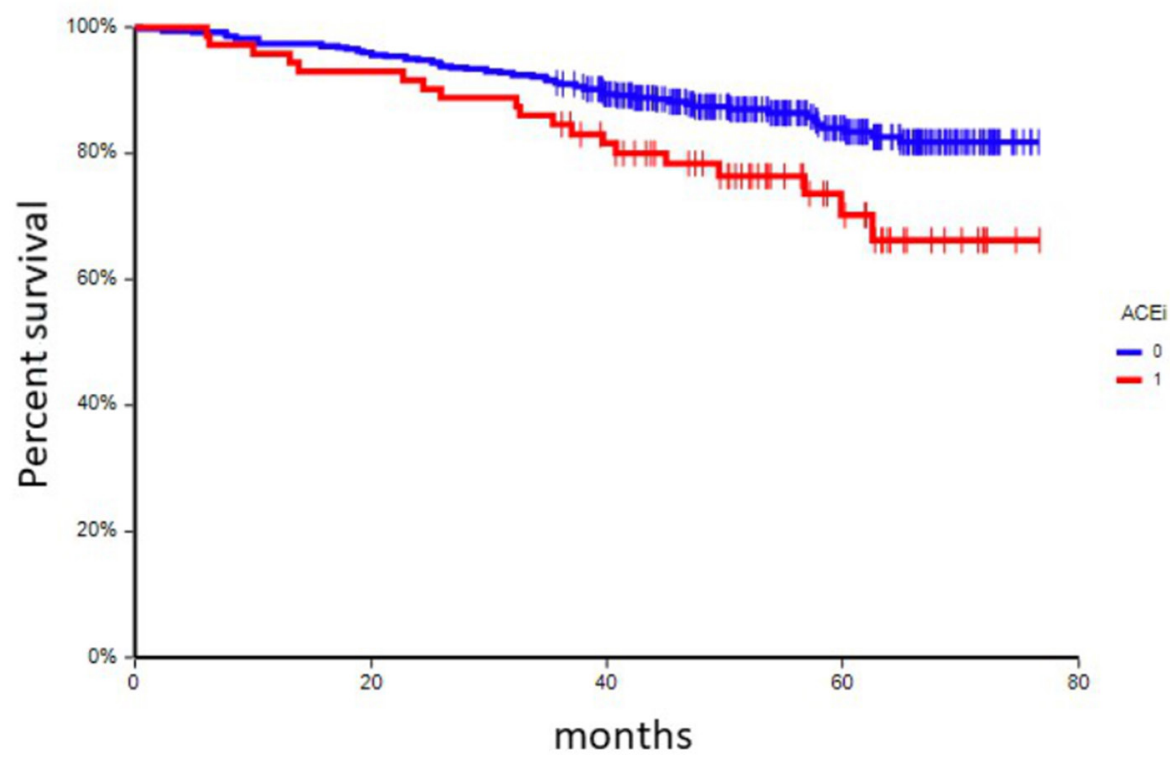

Figure 1. Kaplan-Meier estimates of probabilities of disease-free survival according to chronic administration of ACEi $(n=414), \mathrm{HR}=0.49$, $95 \% \mathrm{Cl} 0.26-0.94, P=0.007$. HR: hazard ratio

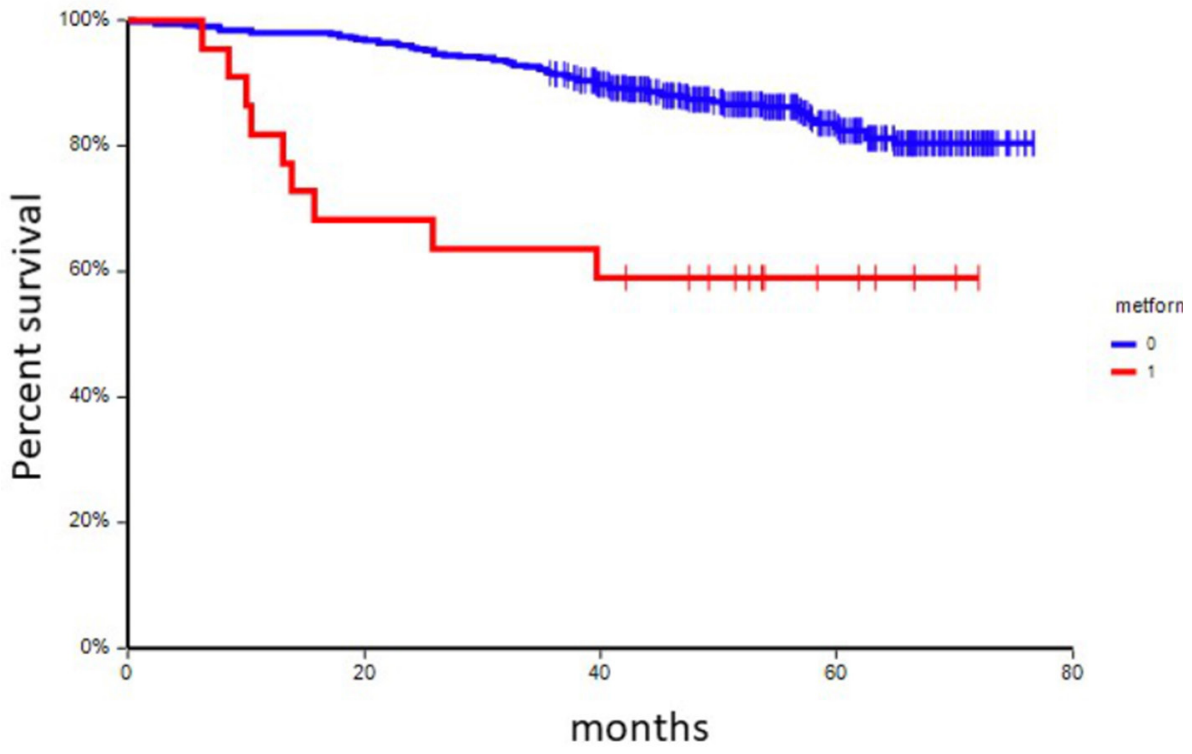

Figure 2. Kaplan-Meier estimates of probabilities of disease-free survival according to chronic administration of metformin $(n=414)$, HR $=0.27,95 \% \mathrm{Cl} 0.08-0.91, P<0.001$. HR: hazard ratio

metformin, and/or insulin was associated with inferior DFS [Table 6 and Figures 1-3] This correlation was most pronounced in patients with CTC_EMT phenotype. The negative prognostic impact of chronic medication was especially observed in patients with CTC_EMT that were on ACEi compared to patients with CTC_EP and/or no CTCs, where administration of ACEi had no impact on patient's prognosis [Table 7 and Figure 4].

In multivariate analysis, presence of CTC_EMT, axillary nodal involvement, hormone receptor status, Ki67 status, and chronic prescription of insulin/metformin were independently associated with DFS [Table 8]. 


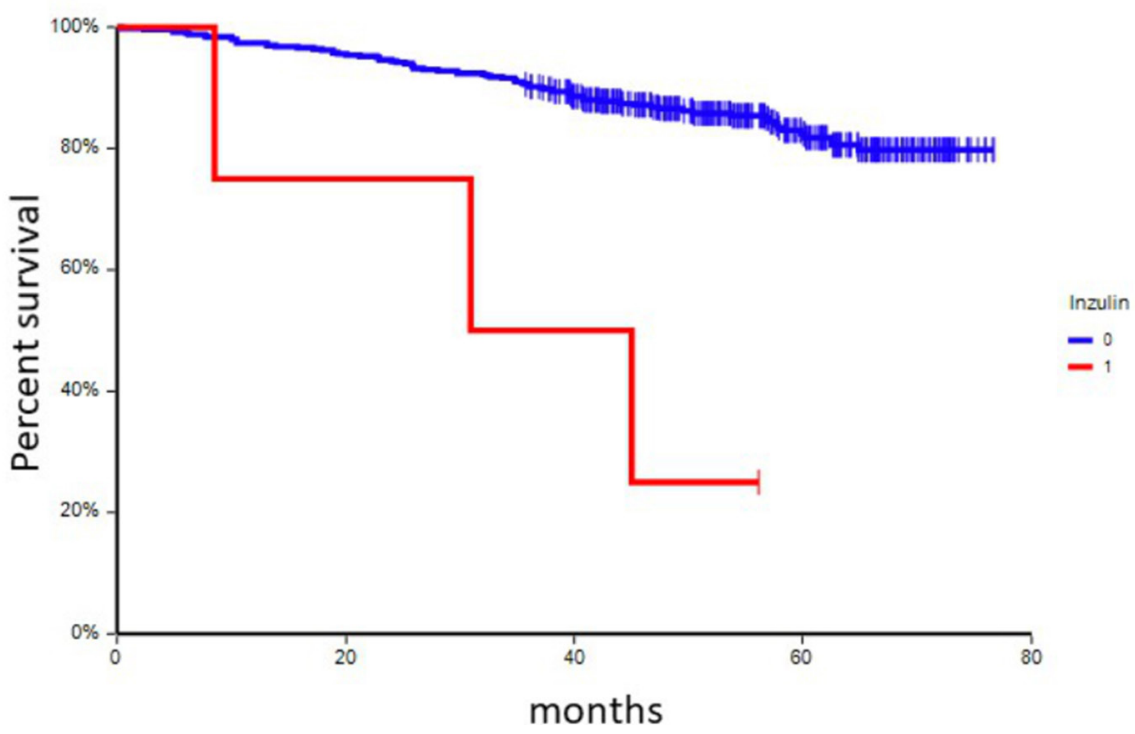

Figure 3. Kaplan-Meier estimates of probabilities of disease-free survival according to chronic administration of insulin $(n=427)$, HR $=$ $0.12,95 \% \mathrm{Cl} 0.01-2.91, P<0.001$. HR: hazard ratio

Table 8. Multivariate analysis of factors associated with disease-free survival

\begin{tabular}{|c|c|c|c|c|}
\hline Variable & HR & 95\% Low & 95\% High & P-value \\
\hline $\mathrm{N}$ stage $\mathrm{N}+v s$. NO & 2.43 & 1.50 & 3.94 & 0.0003 \\
\hline ER/PR status positive for either vs. negative for both & 0.51 & 0.28 & 0.94 & 0.0315 \\
\hline Ki $67>20 \%$ vs. $<20 \%$ & 2.33 & 1.36 & 4.00 & 0.0021 \\
\hline ACEi yes vs. no & 1.71 & 0.94 & 3.08 & 0.0766 \\
\hline Insulin/metformin yes $\mathrm{vs}$. no & 3.97 & 1.95 & 8.08 & 0.0001 \\
\hline CTC_EMT present $v s$. absent & 2.44 & 1.43 & 4.15 & 0.0010 \\
\hline
\end{tabular}

HR: hazard ratio; ER: estrogen receptor; PR: progesteron receptor; CTC_EMT: circulating tumor cell with epithelial-mesenchymal transition phenotype; ACEi: angiotensin-converting-enzyme inhibitors

\section{DISCUSSION}

In this translational study, we observed no association between CTCs, including their different subpopulations and chronic medication, except the trend for association between CTC_EP and ACEi/ sartans, where patients on ACEi/sartans had lower prevalence of CTC_EP compared to patients without ACEi/sartans. We did not noticed association between BMI status and CTCs as well. These data suggest that chronic medication for general co-morbid conditions have only a slight impact on metastatic cascade. Moreover, we observed that chronic administration of ACEi, metformin, and/or insulin was associated with inferior DFS, while, in multivariate analysis, only insulin/metformin remained independently associated with clinical prognosis. CTC status had no effect on patient's outcome according to chronic medication; however, due to small size of several subgroups, e.g., NSAID, low molecular weight heparin, and others, the statistical power for analysis is limited.

Approximately $16 \%$ of breast cancer patients have diabetes ${ }^{[21]}$. Diabetes mellitus not only increases the risk of breast cancer, but might also worsen breast cancer prognosis ${ }^{[21]}$. Insulin resistance and hyperinsulinemia state may be a potential mediator of this effect ${ }^{[22]}$. In our study, chronic use of metformin and insulin was associated with inferior outcome. Previously, it was shown that continual administration of insulin over $\geq 3$ years was associated with an increased risk of mortality in breast cancer ${ }^{[23]}$. Moreover, fasting 
CTCEMT -
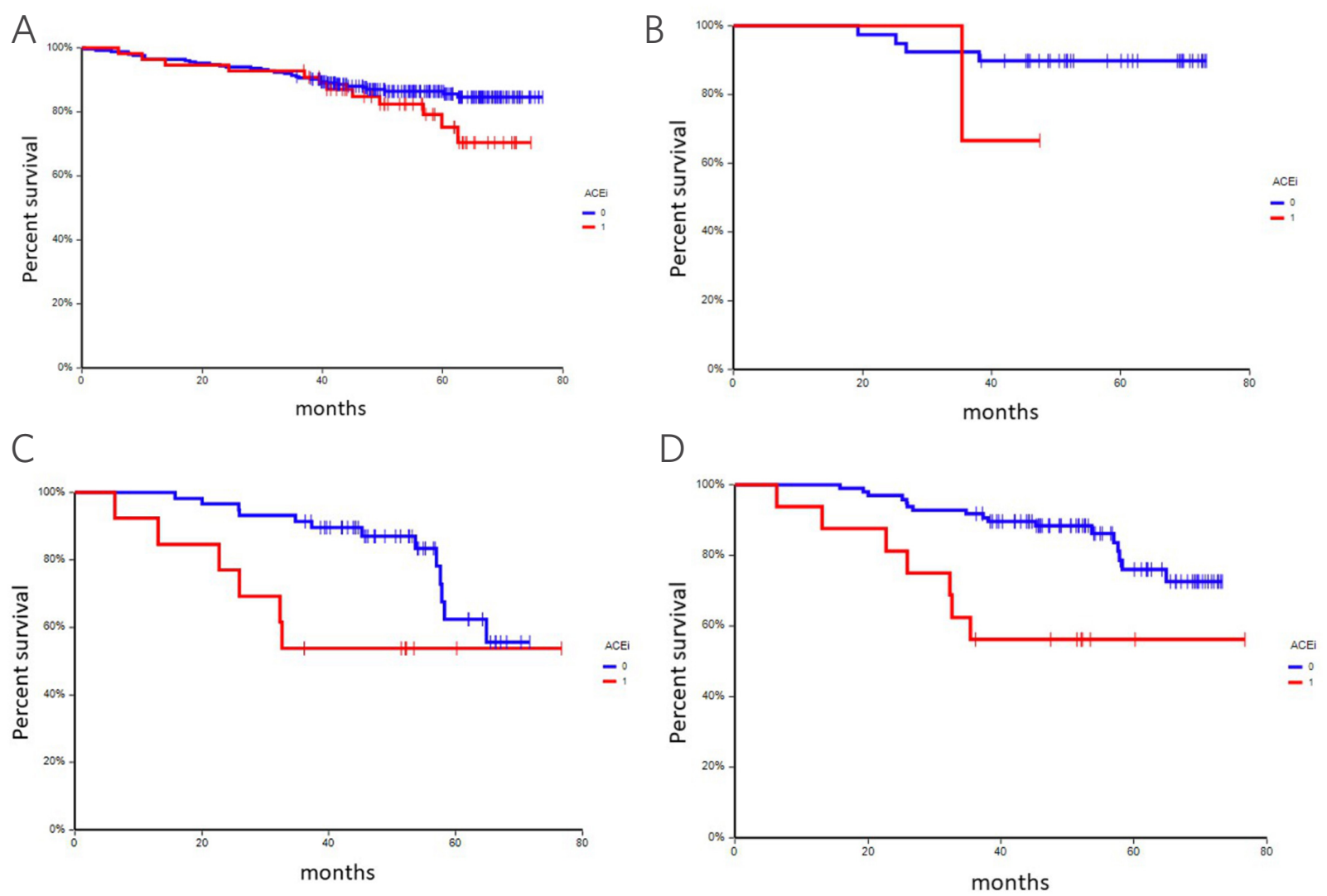

Figure 4. Kaplan-Meier estimates of probabilities of disease-free survival according to CTC status $A, C T C$ negative $B, C T C \_E P$ positive C, CTC_EMT positive D, CTC_any. CTC: circulating tumor cell; CTC_EP: circulating tumor cell with epithelial phenotype; CTC_EMT: circulating tumor cell with epithelial-mesenchymal transition phenotype

hyperinsulinemia was reported to be an independent predictor for higher risk of breast cancer distant recurrence and death in women without known diabetes ${ }^{[24]}$. Insulin is known as an enhancer of cancer cell proliferation, inhibiting apoptosis by its receptor and insulin-like growth factor through the PI3K/Akt and MAPK pathways ${ }^{[2]}$. The other reason contributing to progression of breast cancer is decrease of plasma levels of sex hormone binding globulin related to insulin, which results in increase of endogenous estrogen and androgen levels ${ }^{[22]}$. Contrary to our study, metformin, front-line therapy for the treatment of type 2 diabetes, especially in overweight and obese patients, may reduce breast cancer incidence and improve prognosis by several potential mechanisms according to some preclinical data ${ }^{[24,25]}$. We suppose that, in our trial, worse prognosis associated to metformin and/or insulin administration could be related to diabetes as comorbid condition. Data related to glycemic control and insulinemia were not available. Therefore, we cannot exclude that poor glycemic control and/or hyperinsulinemia might influence this observation. The most negative impact on DFS was observed in CTC_EMT positive subtype. However, our data suggest that worse prognosis related to these drugs might not be related to more efficient metastatic cascade, as there were no differences between CTC and this antidiabetic medication. Due to limited sample size, however, we cannot exclude limited statistical power to definitively answer this question, while the multiple testing approach could affect study results as well. Therefore, our results are only hypothesis generating and validation studies are needed.

Certain classes of antihypertensive drugs are associated with shorter survival in several types of cancers. However, the connection between antihypertensive agents and cancer patient survival remains unclear ${ }^{[2]}$. 
ACEis/angiontensin receptor antagonists are the most active drugs approved for treatment of hypertension, heart failure, and diabetic nephropathy. Several epidemiological studies have investigated relationship between ACEi use and cancer-specific mortality in patients with breast cancer ${ }^{[26-30]}$. In some of them, there was a small increase in cancer recurrence with ACEi use, while others suggest that this drug could be safely administered to breast cancer patients, without affecting breast cancer outcome. In our study, chronic administration of ACEi was associated with inferior DFS, and this was most pronounced in patients with CTC_EMT phenotype. Contrary to this observation, there was no association between ACEi and CTC count.

In conclusion, our findings show that there was no association between chronically used medication and/ or CTCs in patients with primary breast cancer, while chronic administration of ACEi, metformin, and insulin could negatively affect prognosis. These data suggest that evaluated chronic medications are not able to favorably affect biology of primary breast cancer.

\section{DECLARATIONS}

\section{Authors' contributions}

Conception and design of this study: Mardiak J, Mego M

Participated in selection of patients, collection of samples, informed consent obtaining, clinico-pathological data collection: Jurisova S, Karaba M, Benca J, Pindak D

CTCs detection and analysis: Minarik G, Manasova D, Kalavska K, Sedlackova T

Performed statistical analysis: Mego M

Participatedin manuscript preparation: Jurisova S, Mego M

All authors read and approved the final manuscript.

\section{Availability of data and materials}

All data source could be available to readers on request.

\section{Financial support and sponsorship}

This publication is the result of the implementation of projects no. APVV-16-0010 and APVV-14-0327 funded by the Slovak Research and Development Agency.

\section{Conflicts of interest}

All authors declared that there are no conflicts of interest.

\section{Ethical approval and consent to participate}

The study was approved by the Institutional Review Board (IRB) of the National Cancer Institute of Slovakia and all patients signed informed consent.

\section{Consent for publication}

Not applicable.

\section{Copyright}

(c) The Author(s) 2020.

\section{REFERENCES}

1. Bray F, Ferlay J, Soerjomataram I, Siegel RL, Torre LA, et al. Global cancer statistics 2018: GLOBOCAN estimates of incidence and mortality worldwide for 36 cancers in 185 countries. CA Cancer J Clin 2018;68:394-424.

2. Fidler IJ. Metastasis: quantitative analysis of distribution and fate of tumor emboli labeled with 125 I-5-iodo-2'-deoxyuridine. J Natl Cancer Inst 1970;45:773-82. 
3. Aceto N, Bardia A, Miyamoto DT, Donaldson MC, Wittner BS, et al. Circulating tumor cell clusters are oligoclonal precursors of breast cancer metastasis. Cell 2014;158:1110-22.

4. Cristofanilli M, Budd GT, Ellis MJ, Stopeck A, Matera J, et al. Circulating tumor cells, disease progression, and survival in metastatic breast cancer. N Engl J Med 2004;351:781-91.

5. Ignatiadis M, Kallergi G, Ntoulia M, Perraki M, Apostolaki S, et al. Prognostic value of the molecular detection of circulating tumor cells using a multimarker reverse transcription-PCR assay for cytokeratin 19, mammaglobin A, and HER2 in early breast cancer. Clin Cancer Res 2008;14:2593-600.

6. Lucci A, Hall CS, Lodhi AK, Bhattacharyya A, Anderson AE, et al. Circulating tumour cells in non-metastatic breast cancer: a prospective study. Lancet Oncol 2012;13:688-95.

7. Zhang L, Riethdorf S, Wu G, Wang T, Yang K, et al. Meta-analysis of the prognostic value of circulating tumor cells in breast cancer. Clin Cancer Res 2012;18:5701-10.

8. Eliassen AH, Colditz GA, Rosner B, Willett WC, Hankinson SE. Adult weight change and risk of postmenopausal breast cancer. JAMA 2006;296:193-201.

9. Kawai M, Minami Y, Kuriyama S, Kakizaki M, Kakugawa Y, et al. Adiposity, adult weight change and breast cancer risk in postmenopausal Japanese women: the Miyagi Cohort Study. Br J Cancer 2010;103:1443-7.

10. Lahmann PH, Hoffmann K, Allen N, van Gils CH, Khaw KT, et al. Body size and breast cancer risk: findings from the European Prospective Investigation into Cancer And Nutrition (EPIC). Int J Cancer 2004;111:762-71.

11. Ulrich CM, Bigler J, Potter JD. Non-steroidal anti-inflammatory drugs for cancer prevention: promise, perils and pharmacogenetics. Nat Rev Cancer 2006;6:130-40.

12. Alimova IN, Liu B, Fan Z, Edgerton SM, Dillon T, et al. Metformin inhibits breast cancer cell growth, colony formation and induces cell cycle arrest in vitro. Cell Cycle 2009;8:909-15.

13. Baneres Amella J, Alonso Caballero J, Anto Boque JM. The appropriateness of hospital admissions. Med Clin (Barc) 1990;95:357-8.

14. Mu L, Zhu N, Zhang J, Xing F, Li D, et al. Type 2 diabetes, insulin treatment and prognosis of breast cancer. Diabetes Metab Res Rev 2017;33.

15. Mego M, Giordano A, De Giorgi U, Masuda H, Hsu L, et al. Circulating tumor cells in newly diagnosed inflammatory breast cancer. Breast Cancer Res 2015;17:2.

16. Brewer TM, Masuda H, Liu DD, Shen Y, Liu P, et al. Statin use in primary inflammatory breast cancer: a cohort study. Br J Cancer 2013;109:318-24.

17. Mego M, Karaba M, Minarik G, Benca J, Silvia J, et al. Circulating tumor cells with epithelial-to-mesenchymal transition phenotypes associated with inferior outcomes in primary breast cancer. Anticancer Res 2019;39:1829-37.

18. Cierna Z, Mego M, Janega P, Karaba M, Minarik G, et al. Matrix metalloproteinase 1 and circulating tumor cells in early breast cancer. BMC Cancer 2014;14:472.

19. Mego M, Karaba M, Minarik G, Benca J, Sedlackova T, et al. Relationship between circulating tumor cells, blood coagulation, and urokinase-plasminogen-activator system in early breast cancer patients. Breast J 2015;21:155-60.

20. Mego M, Cholujova D, Minarik G, Sedlackova T, Gronesova P, et al. CXCR4-SDF-1 interaction potentially mediates trafficking of circulating tumor cells in primary breast cancer. BMC Cancer 2016;16:127.

21. Peairs KS, Barone BB, Snyder CF, Yeh HC, Stein KB, et al. Diabetes mellitus and breast cancer outcomes: a systematic review and meta-analysis. J Clin Oncol 2011;29:40-6.

22. Wairagu PM, Phan AN, Kim MK, Han J, Kim HW, et al. Insulin priming effect on estradiol-induced breast cancer metabolism and growth. Cancer Biol Ther 2015;16:484-92.

23. Gunter MJ, Hoover DR, Yu H, Wassertheil-Smoller S, Rohan TE, et al. Insulin, insulin-like growth factor-I, and risk of breast cancer in postmenopausal women. J Natl Cancer Inst 2009;101:48-60.

24. Gonzalez-Angulo AM, Meric-Bernstam F. Metformin: a therapeutic opportunity in breast cancer. Clin Cancer Res 2010;16:1695-700.

25. Jiralerspong S, Palla SL, Giordano SH, Meric-Bernstam F, Liedtke C, et al. Metformin and pathologic complete responses to neoadjuvant chemotherapy in diabetic patients with breast cancer. J Clin Oncol 2009;27:3297-302.

26. Chae YK, Brown EN, Lei X, Melhem-Bertrandt A, Giordano SH, et al. Use of ACE inhibitors and angiotensin receptor blockers and primary breast cancer outcomes. J Cancer 2013;4:549-56.

27. Sorensen GV, Ganz PA, Cole SW, Pedersen LA, Sorensen HT, et al. Use of beta-blockers, angiotensin-converting enzyme inhibitors, angiotensin II receptor blockers, and risk of breast cancer recurrence: a Danish nationwide prospective cohort study. J Clin Oncol 2013;31:2265-72.

28. Ganz PA, Habel LA, Weltzien EK, Caan BJ, Cole SW. Examining the influence of beta blockers and ACE inhibitors on the risk for breast cancer recurrence: results from the LACE cohort. Breast Cancer Res Treat 2011;129:549-56.

29. Holmes MD, Hankinson SE, Feskanich D, Chen WY. Beta blockers and angiotensin-converting enzyme inhibitors' purported benefit on breast cancer survival may be explained by aspirin use. Breast Cancer Res Treat 2013;139:507-13.

30. Cardwell CR, Mc Menamin UC, Hicks BM, Hughes C, Cantwell MM, et al. Drugs affecting the renin-angiotensin system and survival from cancer: a population based study of breast, colorectal and prostate cancer patient cohorts. BMC Med 2014;12:28. 